\title{
BMJ Open Impact of ischaemic heart disease severity and age on risk of cardiovascular outcome in diabetes patients in Sweden: a nationwide observational study
}

\author{
Tomas Jernberg, ${ }^{1}$ Daniel Lindholm, ${ }^{2}$ Lars Pål Hasvold, ${ }^{3}$ Bodil Svennblad, ${ }^{2}$ \\ Johan Bodegård, ${ }^{3}$ Karolina Sundell Andersson, ${ }^{4}$ Marcus Thuresson, ${ }^{5}$ \\ David Erlinge, ${ }^{6}$ Magnus Janzon ${ }^{7}$
}

To cite: Jernberg T, Lindholm D, Hasvold LP, et al. Impact of ischaemic heart disease severity and age on risk of cardiovascular outcome in diabetes patients in Sweden: a nationwide observational study. BMJ Open 2019;9:e027199. doi:10.1136/ bmjopen-2018-027199

- Prepublication history and additional material for this paper are available online. To view these files, please visit the journal online (http://dx.doi. org/10.1136/bmjopen-2018027199).

Received 10 0ctober 2018 Revised 25 February 2019 Accepted 27 February 2019

Check for updates

(C) Author(s) (or their employer(s)) 2019. Re-use permitted under CC BY-NC. No commercial re-use. See rights and permissions. Published by BMJ.

For numbered affiliations see end of article.

Correspondence to Lars Pål Hasvold;

paal.hasvold@astrazeneca.com

\section{ABSTRACT}

Objectives To compare short-term cardiovascular (CV) outcome in type 2 diabetes (T2D) patients without ischaemic heart disease (IHD), with IHD but no prior myocardial infarction (MI), and those with prior $\mathrm{Ml}$; and assess the impact on risk of age when initiating first-time glucose-lowering drug (GLD).

Design Cohort study linking morbidity, mortality and medication data from Swedish national registries. Participants First-time users of GLD during 2007-2016. Outcomes Predicted cumulative incidence for the $\mathrm{CV}$ outcome (MI, stroke and CV mortality) was estimated. A Cox model was developed where age at GLD start and CV risk was modelled.

Results 260070 first-time GLD users were included, 221 226 (85\%) had no IHD, 16294 (6\%) had stable IHD—prior MI and $22550(9 \%)$ had IHD+MI. T2D patients without IHD had a lower risk of CV outcome compared with the IHD populations ( \pm prior MI), (3-year incidence $4.78 \%$ vs $5.85 \%$ and $8.04 \%$ ). The difference in CV outcome was primarily driven by a relative greater Ml risk among the IHD patients. For T2D patients without IHD, an almost linear association between age at start of GLD and relative risk was observed, whereas in IHD patients, the younger ( $<60$ years) patients had a relative greater risk compared with older patients.

Conclusions T2D patients without IHD had a lower risk of the CV outcome compared with the T2D populations with IHD, primarily driven by a greater risk of MI. For T2D patients without IHD, an almost linear association between age at start of GLD and relative risk was observed, whereas in IHD patients, the younger patients had a relative greater risk compared with older patients. Our findings suggest that intense risk prevention should be the key strategy in the management of T2D patients, especially for younger patients.

\section{BACKGROUND}

In recent years, there has been an increase in the incidence of diagnosed type 2 diabetes (T2D) in both developed and developing
Strengths and limitations of this study

- The study was conducted in a nationwide national cohort including all patients who collected a firsttime glucose-lowering drug (GLD) at the pharmacy during the observational period, limiting the potential problems with selection bias.

- Access to clinical data describing the type 2 diabetes (T2D) duration (prior to GLD therapy), extent and severity of T2D (blood glucose, $\mathrm{HbA}_{1 \mathrm{c}}$, weight, smoking pattern and kidney function) which have an impact on the risk was not available.

- The study is reliant on International Statistical Classification of Diseases and Related Health Problems, 10th Revision codes for morbidity data and therefore, the possibility of coding errors cannot be ruled out.

- Another limitation of our study was the lack of available data on socioeconomic status which is known to affect risk in T2D patients.

societies, with approximately 425 million people $(8 \%-9 \%)$ living with diabetes worldwide in $2017 .^{1}$

T2D is commonly associated with macrovascular complications, often resulting in early manifestation of ischaemic heart disease (IHD) and increased risk of cerebrovascular disease. ${ }^{2}$ Diabetes is associated with a substantial increase in risk of major cardiovascular (CV) events in patients both with and without established cardiovascular disease (CVD) ${ }^{3-7}$ This to such extent that CVD-related death is the most common cause of mortality among diabetes patients. ${ }^{8}$

There are several theories for why T2D patients have increased CV risk, a common view is that increased hyperglycaemic stress may worsen the prognosis in T2D patients 
and that T2D patients may be exposed to plaque instability due to the pro-inflammatory/oxidative properties of their plaque. ${ }^{910}$ This may result in a more severe IHD among T2D patients, as it is well known that T2D patients often have coronary multivessel disease and often have more severe CV outcome than other IHD patients. ${ }^{11}$

There has been an improvement in post-myocardial infarction (MI) survival in Western countries, leading to an overall growth of the population with a history of IHD. ${ }^{12}$ Combined with the increased incidence of T2D, it is likely that the T2D patient population with a history of IHD will increase in the coming decades and thus, increased knowledge of the short-term CV event pattern is important.

So far, there are no studies comparing the short-term prognostic impact of a history of clinical stable IHD with that of an atherothrombotic disease demonstrated as previous MI in diabetic patients. Moreover, the consequences of age when initiating glucose-lowering drug (GLD) in relation to short-term GVD risk have not been well described either. These are all important considerations when targeting patients for intensified secondary preventive measures.

The primary objective of the present study was to compare short-term (3-year) CV outcome in T2D patients without IHD, with IHD but no prior MI, and those with prior MI. Secondary objectives were to assess the impact of age when initiating first-time GLD. For these purposes, we used a highly representative nationwide sample of all T2D patients initiating first-time GLD in Sweden over 7 years.

\section{METHOD}

\section{Study design}

Data for this observational study were retrieved by linking data from Swedish mandatory nationwide registers: the Swedish National Patient Register (NPR; including inpatient admission and discharge dates, and main and secondary diagnoses according to the International Statistical Classification of Diseases and Related Health Problems, 10th Revision [ICD-10]), the Swedish Prescribed Drug Register (SPDR) ${ }^{13}$ and the Swedish Cause of Death Register. ${ }^{14}$ The Swedish NPR has had mandatory registration since 1984 and covers more than $99 \%$ of all somatic (including surgery) and psychiatric hospital admissions and discharges. ${ }^{15}$ The SPDR contains data on all prescribed medications collected from pharmacies in Sweden since 2005. Linkage of patient-level data was performed by the Swedish National Board of Health and Welfare using the unique personal identification (ID) numbers, mandatory for every citizen in Sweden, and thereafter replaced by a study ID for further data processing.

\section{Study population}

The study population included all patients with T2D initiating use of GLD (ATC code A10B) from 1 January 2007 to 31 December 2016. The index date was defined as the date of the first collected prescription of a GLD from the pharmacy during the observation period. To be defined as a first-time user, the patient should not have collected any prescriptions for GLD prior to the index date. Patient characteristics at baseline were established using hospitalisation (ICD-10 diagnoses codes) and drug utilisation data from national registers from 1987 onwards. Patients with collection of GLD from the pharmacy before the study period were excluded.

Three study sub populations were defined based on patients' clinical characteristics when collecting their first-time GLD was from the pharmacy (baseline/index).

1. T2D patients without IHD: first-time GLD treated T2D patients without any previous diagnosis of IHD (defined as a history of MI, unstable angina or stable angina pectoris) (ICD-10: I20-I25).

2. T2D patients with IHD without prior MI: first-time GLD treated T2D patients with previous diagnosis of IHD without MI (defined as a history of stable or unstable angina pectoris [ICD-10: I25], but no MI).

3. T2D patients with IHD with prior MI: defined as firsttime GLD treated T2D patients with a history of MI (ICD-10: I21-I24).

For the secondary objective, the three study populations were further stratified into the following age categories (based on age at first GLD collection GLD) : $<55$ years, $55-64$ years, $65-74$ years, $75-84$ years and $>85$ years.

\section{Outcomes}

The primary CV outcome was a composite of hospitalisation with main diagnosis of non-fatal MI (ICD-10: I21), non-fatal stroke (ICD-10: I61-I64) or CV death (death with ICD-10 codes I00-I99 as a primary diagnosis).

\section{Sensitivity and additional analyses}

In order to test the impact of prior stroke, outcomes in T2D patients without IHD, MI or stroke were compared with T2D patients with IHD without prior MI or stroke, and T2D patients with IHD with MI without stroke.

All-cause mortality in the three study populations was described.

\section{Patient and public involvement}

This was a cohort study using nationwide register data. No patients were involved in the design of the study. The presented results will hopefully lead to an increased awareness of $\mathrm{CV}$ risk for various T2D populations and thus lead to improved management of patients with T2D.

\section{Statistical analyses}

Baseline characteristics are presented as mean and SD for continuous variables and absolute and relative frequencies for categorical variables. Each patient was followed from date of index date to date of death, or end of study observational period. Comparison between groups with respect to time to event outcomes were analysed using Cox proportional hazards models adjusted for age, sex, T2D duration, atrial fibrillation and heart failure. The results are illustrated using predicted cumulative 
incidence plots (based on the Cox models), as well as in unadjusted Kaplan-Meier plots.

In order to explore the change in relative risk related to age, a Cox model was developed where age was modelled using a restricted cubic spline with five knots. The results are illustrated as the log of the HR over time with the mean age of the total cohort as the reference.

Results are presented as HRs and 95\% CIs. Statistical analyses were performed using SAS V.9.3 and R V.3.5.0.

\section{RESULTS}

Overall, 260070 T2D patients were first-time users of a GLD during the observation period and could be included. Of these, 221226 (85\%) were included in T2D population without IHD, $16294(6 \%)$ in the T2D IHD population without MI and $22550(9 \%)$ in the T2D IHD population with a history of MI. Mean follow-up (FU) was 4.5 years with a maximum of 9.0 years, compromising a total of 1179802 patient years of FU.

T2D patients without IHD were younger (61.4 years), more often female $(45 \%)$ and had a lower incidence of stroke $(5 \%)$, atrial fibrillation $(6 \%)$ and heart failure (3\%) compared with the two T2D IHD populations (table 1). The IHD population without a history of MI had a mean age of 70.9 versus 70.3 years in IHD patients with a history of MI. The two IHD populations included $40 \%$ versus $29 \%$ women, $10 \%$ versus $11 \%$ stroke, $22 \%$ versus $22 \%$ atrial fibrillation and $19 \%$ versus $28 \%$ heart failure, respectively. There were minor differences in GLD therapy among the three study populations, with the majority of patients treated with metformin $(>76 \%)$, sulfonylurea $(>6 \%)$ or insulin $(>11 \%)$. A greater proportion of the T2D IHD population with a history of MI were treated with insulin $(15.0 \%)$. More patients in the two IHD populations were treated with statins ( $68 \%$ vs $26 \%)$, antiplatelets ( $71 \%$ vs $17 \%$ ) and antihypertensives (92\% vs $56 \%$ ) than the T2D patients without IHD.

The cumulative rate of the primary composite $\mathrm{CV}$ outcome (MI, stroke or CVD) was 5.69\% in T2D population without IHD, $13.08 \%$ in the T2D IHD population without MI and $18.83 \%$ in the T2D IHD population with a history of MI during the 3 years FU (see online supplementary figure 1). T2D patients without IHD had a lower risk of the $\mathrm{CV}$ outcome compared with the T2D populations with IHD (3-year adjusted cumulative incidence for a 63-year-old patient [mean age of the study population] $4.78 \%$ vs $5.85 \%$ and $8.04 \%$ ) (figure 1 and online supplementary table 1A). The greater risk seen for T2D IHD patients with no prior MI versus patients without IHD was primarily driven by MI (3-year adjusted cumulative incidence $1.66 \%$ vs $3.09 \%$ ) (figure 1 and online supplementary table 1B). The risk for stroke and CV death followed the same pattern as did the primary CV outcome and MI (see online supplementary table $1 \mathrm{C}, \mathrm{D}$ ). The 3 years cumulative mortality rate was $8.08 \%, 14.77 \%$ and $18.68 \%$ in the three study groups (see online supplementary figure 2 ).
The results of the sensitivity analysis with exclusion of patients with a history of stroke showed a consistent pattern to the main results, T2D patients without IHD or stroke, had a lower risk of CV outcome compared with T2D IHD patients without MI and stroke and T2D patients with MI without stroke (3-year adjusted cumulative incidence for a 62-year-old patient [mean age of the sensitivity analysis population] $4.22 \%$ vs $5.28 \%$ and $7.80 \%)$. Also, in this population, the difference in risk was primarily driven by MI.

The baseline characteristics for patients were stratified by age at first pharmacy GLD collection irrespective of IHD status during the observation period (table 2). The proportion of women was lower, in the younger categories ( $<55$ years), $42 \%$ compared with $61 \%$ among patients older than 85 years. The proportion of patients with cardiovascular comorbidities was greater in older patients, the proportion of patients with previous MI in $>85$ years was $19 \%$, compared with $3 \%$ in patients $<55$ years, and the corresponding numbers for heart failure were $26 \%$ and $1 \%$, respectively. The increased CV burden among the older patients was also reflected in the proportion of patients treated with secondary preventive drugs; the proportion of patients on statin therapy increased from $16 \%$ to $26 \%$ and for antiplatelets from $8 \%$ to $53 \%$ for patients $<55$ years to $>85$ years. A larger proportion of the older patients were treated with insulin and sulfonylurea, whereas younger patients predominately were treated with metformin.

The short-term (3-year after index) risk of CV outcome for T2D patients differed among the three study populations in relation to age when collecting first GLD from pharmacy. Patients without IHD showed an almost linear association between age and relative risk of CV outcome respectively (figures 2 and 3). The presence of IHD was associated with a relatively higher increase in relative risk of $\mathrm{CV}$ outcome in younger ( $<60$ years) patients (figures 2 and 3). In patients with IHD, with or without previous $\mathrm{MI}$, the relative $\mathrm{CV}$ and $\mathrm{MI}$ risk did not increase with age in patients younger than 65 years. In patients older 65 years, there was an increased relative risk of CV outcome and risk of MI with increasing age (figures 2 and 3 ). In all age categories, the MI risk was the main risk contributor, both for T2D patients without IHD or MI and for the two IHD populations.

\section{DISCUSSION}

In the present study, we examined a highly representative sample of all T2D patients initiating first-time glucose lowering treatment in a whole country for an 8-year period. There were two key findings: first, T2D patients without IHD had a lower risk of the CV outcome compared with the T2D populations with IHD. The difference in CV outcome was primarily driven by a greater risk of $\mathrm{MI}$ in T2D patients with IHD. Second, for T2D patients without IHD an almost linear association between age at start of GLD therapy and relative risk of CV outcome and MI 
Table 1 Baseline demographic and clinical characteristics for first-time GLD treated T2D patients without IHD, T2D IHD patients without $\mathrm{MI}$ and in T2D IHD patients with a history of MI

\begin{tabular}{|c|c|c|c|c|}
\hline & No IHD $n=221226$ & IHD without MI n=16 294 & IHD with MI n=22 550 & Total $n=260070$ \\
\hline Age (years, mean, SD) & $61.4(13.8)$ & $70.9(10.5)$ & $70.3(11.1)$ & $62.8(13.8)$ \\
\hline \multicolumn{5}{|l|}{ Age group (years) } \\
\hline $55-64, \mathrm{n}(\%)$ & $60785(27.5)$ & 3480 (21.4) & 4944 (21.9) & $69209(26.6)$ \\
\hline $65-74, \mathrm{n}(\%)$ & $57619(26.0)$ & $5671(34.8)$ & 7484 (33.2) & 70774 (27.2) \\
\hline Female sex, n (\%) & 99389 (44.9) & 6472 (39.7) & $6632(29.4)$ & $112493(43.3)$ \\
\hline Myocardial infarction, n (\%) & $0(0.0)$ & $0(0.0)$ & $22550(100.0)$ & $22550(8.7)$ \\
\hline Unstable angina, n (\%) & $0(0.0)$ & $3286(20.2)$ & $5085(22.5)$ & $8371(3.2)$ \\
\hline Angina pectoris, $\mathrm{n}(\%)$ & $0(0.0)$ & 8014 (49.2) & $6545(29.0)$ & $14559(5.6)$ \\
\hline Heart failure, n (\%) & $7612(3.4)$ & $3025(18.6)$ & $6386(28.3)$ & $17023(6.5)$ \\
\hline \multicolumn{5}{|c|}{ Prescribed drugs at first collection of glucose lowering drug } \\
\hline Antiplatelets, $\mathrm{n}(\%)$ & $37214(16.8)$ & $11670(71.6)$ & $18081(80.2)$ & $66965(25.7)$ \\
\hline Clopidogrel, n (\%) & $1781(0.8)$ & $1312(8.1)$ & $3716(16.5)$ & $6809(2.6)$ \\
\hline Low-dose ASA, n (\%) & $35797(16.2)$ & $11248(69.0)$ & 17407 (77.2) & $64452(24.8)$ \\
\hline Anticoagulants, n (\%) & $10636(4.8)$ & $2376(14.6)$ & $2945(13.1)$ & $15957(6.1)$ \\
\hline Statins, $n(\%)$ & $58288(26.3)$ & $11146(68.4)$ & $17160(76.1)$ & $594(33.3)$ \\
\hline Antihypertensives, n (\%) & $122861(55.5)$ & $14962(91.8)$ & $20805(92.3)$ & $158628(61.0)$ \\
\hline Beta-blockers, n (\%) & 61174 (27.7) & 11774 (72.3) & 18098 (80.3) & 91046 (35.0) \\
\hline Insulin, n (\%) & 25181 (11.4) & 1917 (11.8) & $3384(15.0)$ & 30482 (11.7) \\
\hline Metformin, n (\%) & 185387 (83.8) & 12995 (79.8) & 17211 (76.3) & 215593 (82.9) \\
\hline SU, n (\%) & 13049 (5.9) & $1311(8.0)$ & $1899(8.4)$ & 16259 (6.3) \\
\hline DPP-4is, n (\%) & $1784(0.8)$ & $181(1.1)$ & $327(1.5)$ & $2292(0.9)$ \\
\hline Metiglinides, n (\%) & $2488(1.1)$ & $274(1.7)$ & $430(1.9)$ & $3192(1.2)$ \\
\hline
\end{tabular}

ACEI, angiotensin-converting enzyme inhibitor; ARB, angiotensin receptor blocker; ASA, acetylsalicylic acid; Ca-blockers, calciumchannel blocker; DPP-4is, pipeptidyl peptidase-4 inhibitors; GLD, glucose lowering drug; SU, sulfonylurea.

was observed, whereas in IHD patients, the younger $(<60$ years) patients had a relative greater increase in shortterm CV risk compared with older patients. Also, in T2D patients with IHD, there was no increase in relative $\mathrm{CV}$ risk with increasing age until the age of 65 , with a linear increase in risk thereafter.

Previously, it has been shown that a T2D population compared with the general population in Sweden has significant increased risk for $\mathrm{CV}$ events like MI, heart failure, atrial fibrillation and all-cause death. ${ }^{16}$ Over time from 1998 to 2013, the incidence of hospitalisation for
CVD and CV mortality has almost decreased by half in patients with T2D but remained considerably higher than in matched controls without T2D. ${ }^{17}$

Our findings highlight that there is a marked difference in $\mathrm{CV}$ and MI risk in different T2D populations related to the presence and severity of IHD disease. Even the T2D patients without prior MI have a risk that is comparable to a post MI population. ${ }^{12}$ Bearing in mind that we focus on difference in short-term risk in this paper (3 year after initiation of GLD treatment for T2D), and still see large differences in risk between the different study populations, it is 


\section{— No IHD $\quad-$ - IHD wo MI -.- IHD with MI}
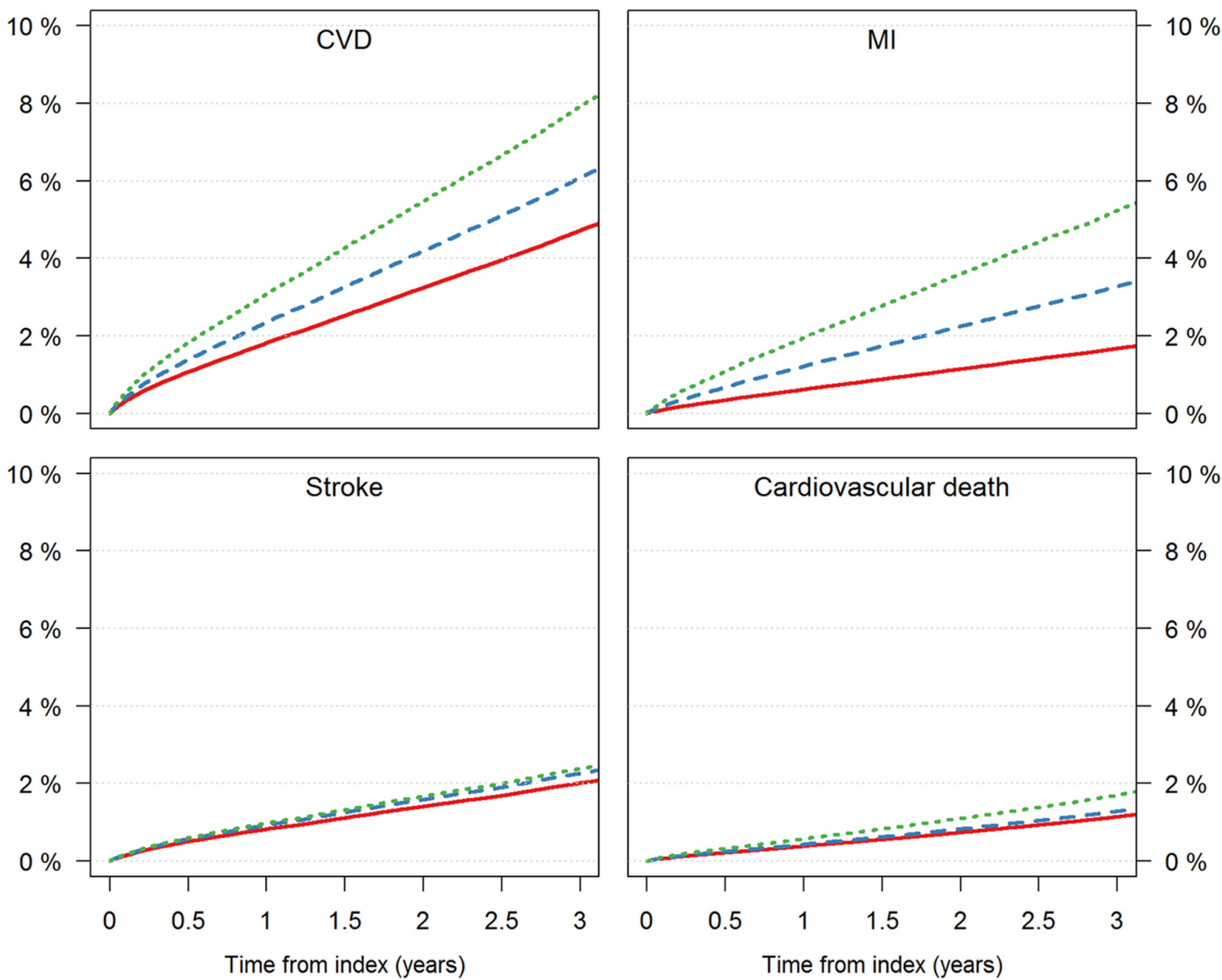

Figure 1 Adjusted probability plots ${ }^{\star}$ for time to the first occurrence of the composite CV composite outcome, and the components MI, stroke and CV death separately among T2D patients without IHD, T2D IHD patients without MI and in T2D IHD patients with a history of Ml.*Predicted for the 'average' 63-year-old patient (mean age of the study population, and 'mean' of the following risk factors: sex, diabetes duration, atrial fibrillation and heart failure). CV, cardiovascular; CVD, cardiovascular disease; IHD, ischaemic heart disease; MI, myocardial infarction; T2D, type 2 diabetes.

inevitable that T2D patients with a history of IHD should be carefully monitored and managed with a long-term perspective. That is, by effective prevention programmes and aggressive drug therapy after being diagnosed with T2D, particularly in those considered to be at high risk of ischaemic events. The importance of risk factor control has recently been shown in a study from the Swedish National Diabetes Registry (NDR) where patients with T2DM who had appropriate risk factor control had little or no excess risk of $\mathrm{CV}$ events as compared with the general population. ${ }^{18}$

The data included in our study is recent, but as management of T2D is rapidly progressing, the observed GLD therapy, with a high proportion of patients treated with insulin. T2D drug therapy with the novel drug options, including glucagon-like peptide 1 analogues and sodium-glucose cotransporter 2 inhibitors, have in addition to improving glucose control, been shown to be associated with lower rates of $\mathrm{CV}$ events and mortality compared with older glucose lowering therapies. ${ }^{19-22}$
Only a limited number of studies have examined the association between age at start of first-time GLD and CV risk. A recent study from Australia showed that a younger age at T2D diagnosis was associated with a higher risk of all-cause and CVD-related death. ${ }^{23}$ Hence, the duration of T2D was shown to be an important factor for life-time risk, as also reported previously in several other publications. ${ }^{16}$ That said, there is still a scarcity of data describing CV risk in T2D age-stratified populations, comparable to our findings, focusing on the relatively short-term CV risk after start of GLD therapy, and not the full life-time risk in relation to age at start of GLD therapy. Our findings are based on time from first collection of GLD from the pharmacy and not the total duration of T2D prior to index (start of GLD therapy). We can thus only speculate that the IHD populations in general are older, have a higher morbidity burden and consequently would have seen more frequently by healthcare professionals and thus might have a relative earlier detection of their T2D than the non-IHD patients. ${ }^{24}$ The Swedish NDR has reported 


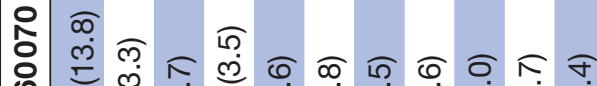
ஸ $\infty$ 过

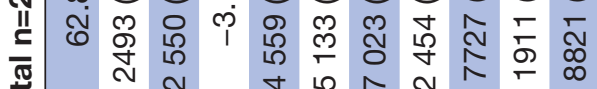

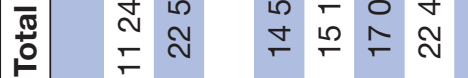

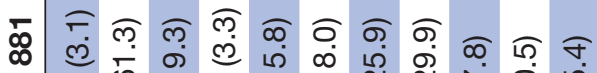

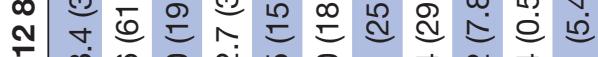

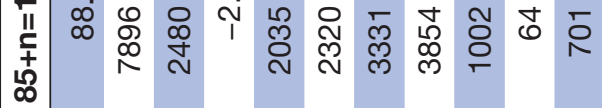

लె

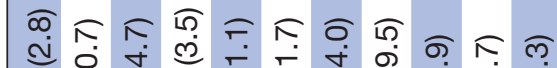

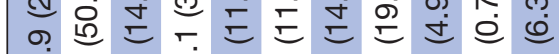
呮 م

त

क्ष

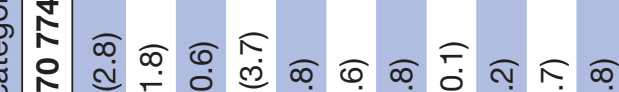

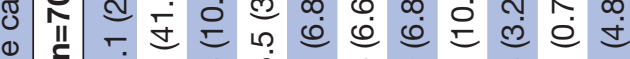
范 वे

$\frac{\pi}{\frac{\pi}{5}}$

产

产

$\stackrel{0}{ᄃ}$

흐 छ

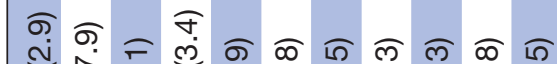

の ल

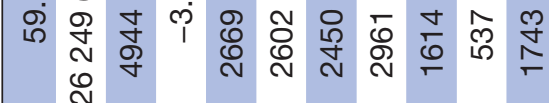
¿

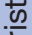

$\frac{\sqrt{2}}{\frac{10}{2}}$

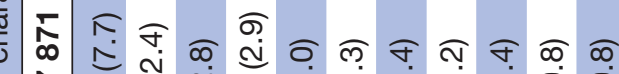

ชู

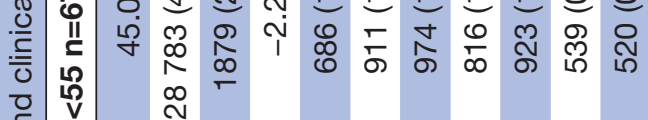

을

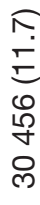

ก

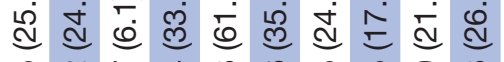
นึ กิ กิ ๑

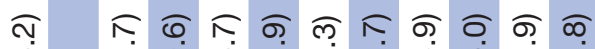
@ 

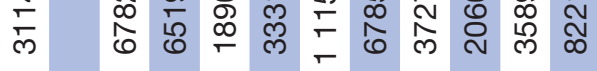

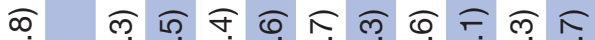

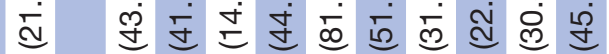
$\infty$ N $m$ 잉 $\infty$ ल 0 फ

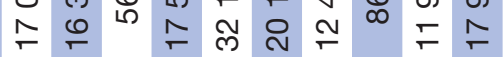

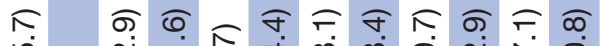
ம の ㄷำ กิ

ปे

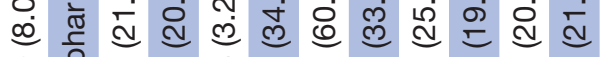
윰유 이 ᄚ

吕 0 ㅇํㅁ

$\sum_{\substack{\frac{1}{2} \\ \frac{1}{2}}}^{0}$

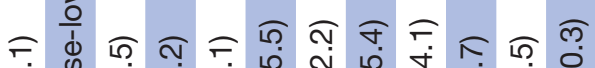
๗் 들

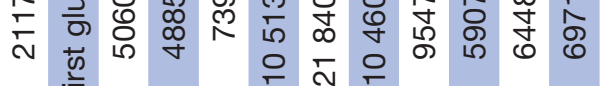

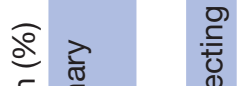

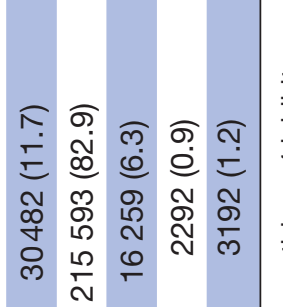

$\frac{w}{<}$

8

然

$\frac{\bar{D}}{\vec{D}}$

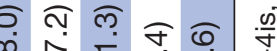

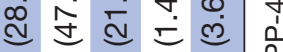

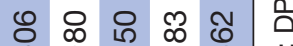

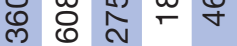

ले ले

ช

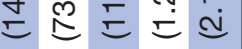

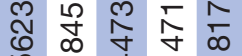

舟

ธุ ल क

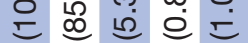
m 员众的

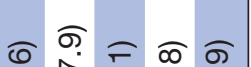

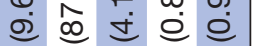
암 뉴 하

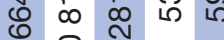
0 氺

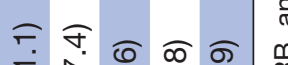
Е क् ल্

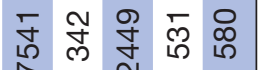
के ले एक

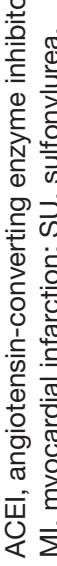




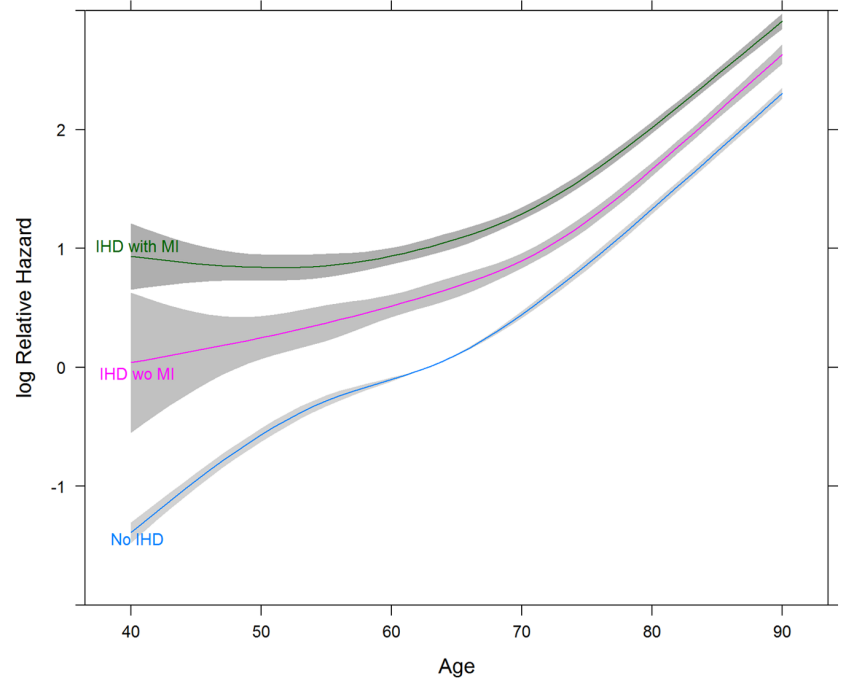

Figure 2 Spline plots for risk of composite CV outcome by age and IHD severity. Reference is mean age (63 years) in the T2D no IHD population. No IHD: T2D patients without IHD, T2D patients without any previous diagnosis of IHD. IHD wo MI: T2D patients with IHD without prior MI, T2D patients with previous diagnosis of IHD without MI. IHD with MI: T2D patients with IHD with prior MI, defined as T2D patients with a history of MI, and unstable angina or angina pectoris. CV, cardiovascular; IHD, ischaemic heart disease; MI, myocardial infarction; T2D, type 2 disease.

that time from T2D diagnosis to GLD therapy initiation has been shorten during 2002 to $2011 .^{25}$

For patients without a history of IHD at start of GLD therapy, an almost linear association between age and

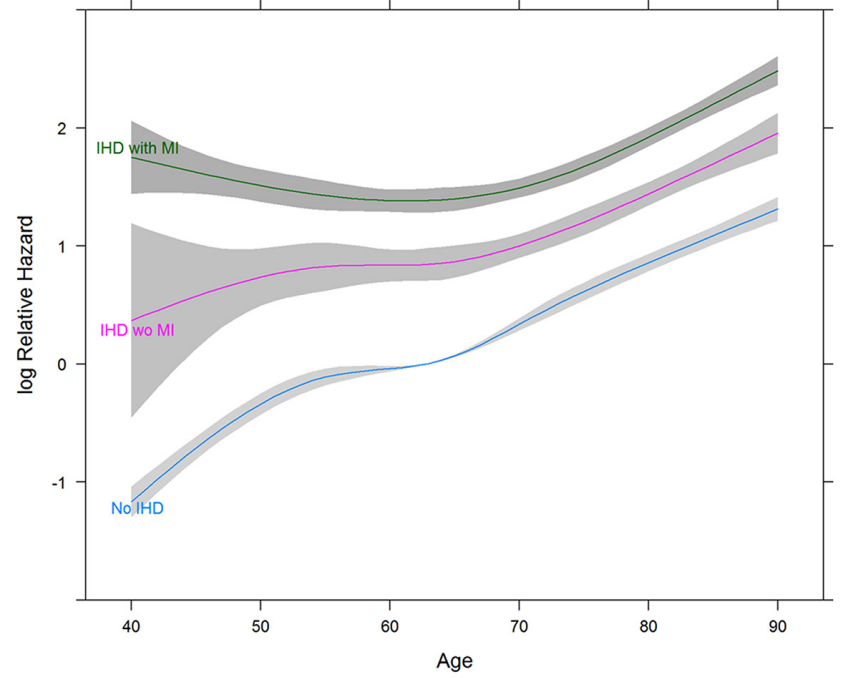

Figure 3 Spline plots for MI risk by age and IHD severity. Reference is mean age (63 years) of the T2D no IHD population. No IHD: T2D patients without IHD, T2D patients without any previous diagnosis of IHD. IHD wo MI: T2D patients with IHD without prior MI, T2D patients with previous diagnosis of IHD without MI. IHD with MI: T2D patients with IHD with prior MI, defined as T2D patients with a history of $\mathrm{MI}$, and unstable angina or angina pectoris. IHD, ischaemic heart disease; MI, myocardial infarction; T2D, type 2 disease.
$\mathrm{CV}$ and MI risk was observed. In contrast, a relatively higher increase in risk for $\mathrm{CV}$ outcome and MI events was observed in younger patients with established IHD when starting with GLD, compared with older patients with IHD. Furthermore, a low proportion of the patients were treated with statins and antiplatelets, ranging from $16 \%$ statins and $8 \%$ antiplatelets for patients below 55 years, to $45 \%$ statins and $43 \%$ antiplatelets for patients of $75-84$ years.

Recent data from Sweden complementing our data examined clinical characteristics in age stratified T2D patients, and showed that patients who develop T2D earlier in life are more frequently obese, have a more adverse lipid profile, higher $\mathrm{HbA}_{1 \mathrm{c}}$ levels and a faster deterioration in glycaemic control compared with individuals who develop diabetes later in life. ${ }^{26}$ They also found that a low proportion of these young patients received blood pressure lowering drugs, statins and antiplatelet drugs. ${ }^{26}$ This more severe metabolic dysregulation, and inadequately provided drug therapy seen, may be associated with accelerated atherosclerosis, supported by the recent findings of a correlation between younger age at T2D diagnosis, increasing number of variables not within target ranges and a higher relative risk of CVD outcomes. ${ }^{18}$ The commonly seen low socioeconomic status among younger T2D patients might be a contributing factor to the noted increased risk, as low socioeconomic status in itself increases risk among T2D patients. ${ }^{27}{ }^{28}$ This should be amenable to primary prevention both by lifestyle changes and medical treatment.

The combination of metabolic risk factors, start of GLD therapy and presences of IHD in young age predicts high risk of major adverse CV events, especially risk for MI. These findings further highlight the importance of providing a closer monitoring of younger T2D patients with established IHD. This is also further highlighted by our findings, where younger T2D patients in general are not receiving adequate treatment with blood pressure lowering drugs, statins and antiplatelet therapy. That said, there may be a possible uncertainty regarding clinical responsibility for drug treatment initiation between specialist and primary care for these patients, which may hamper a thorough T2D patient management.

The strengths of our study are that it was conducted in a nationwide national cohort including all patients collecting a first-time GLD from pharmacy during the observational period, which limits the potential problems with selection bias. The study however also has limitations. First, we did not have access to clinical data describing the duration of T2D prior to GLD start, extent and severity of T2D (blood glucose, $\mathrm{HbA}_{1 \mathrm{c}}$, weight, smoking pattern and kidney function) which have an impact on the risk. ${ }^{29} 30$ However, complementary data on these clinical variables from Sweden were recently published, showing that an unfavourable metabolic profile of the younger patients could be a part of the explanation for our findings. ${ }^{26}$ Second, our study is reliant on ICD-10 codes for morbidity data and therefore, the possibility of coding errors cannot 
be ruled out. However, previous data show that coding is correct in $98 \%$ of Swedish NPR entries. ${ }^{15}$ Another limitation of our study was the lack of available data on socioeconomic status which is known to affect risk in T2D and CAD patients. ${ }^{31}$

\section{CONCLUSION}

In conclusion, T2D patients without IHD had a lower risk of the CV outcome compared with the T2D populations with IHD. The difference in CV outcome was primarily driven by a relative greater MI risk among the T2D IHD patients. Younger ( $<60$ years $)$ T2D IHD patients had a relatively higher risk of $\mathrm{CV}$ outcome, with $\mathrm{MI}$ as the main risk driver, compared with the older T2D IHD patients and T2D patients without a history of IHD. Risk factors other than age and conventional $\mathrm{CV}$ comorbidities seemed to be more important in T2D IHD patients below the age of 60 years at diagnosis, compared with older T2D IHD patients.

Our findings suggest that intense risk prevention should be the key strategy in the management of T2D patients, especially for younger patients, including both encouragement for positive lifestyle changes and prescription of secondary preventive drug therapy with antiplatelet therapy and statins. Ideally, to reduce CV outcome and progression of T2D, younger T2D patients with IHD should be offered participation in guideline-recommended risk reduction programmes.

\section{Author affiliations}

${ }^{1}$ Department of Clinical Sciences, Danderyd University Hospital, Karolinska Institute, Stockholm, Sweden

${ }^{2}$ UCR-Uppsala Clinical Research center, Uppsala Clinical Research center, Uppsala, Sweden

${ }^{3}$ Medical Department, AstraZeneca Nordic, Oslo, Norway

${ }^{4}$ Global Medical Affairs CardioVascular, Renal and Metabolic, AstraZeneca R\&D,

Gothenburg, Sweden

${ }^{5}$ Statisticon, Stockholm, Sweden

${ }^{6}$ Clinical Science, Lunds Universitet, Lund, Sweden

${ }^{7}$ Cardiology, Linkopings Universitet, Linkoping, Sweden

Acknowledgements The authors would like to thank Urban Olsson, Statisticon AB, for data management.

Contributors Data collection was performed by JB. Statistical analysis was conducted by TJ, LPH and MT. Analysis, interpretation and drafting of the manuscript were conducted by TJ and LPH in cooperation with DL, BS, JB, KSA, MT, DE and MJ. All authors approved the manuscript before submission.

Funding The study was sponsored by AstraZeneca.

Competing interests LPH, DL, JB and KSA are employed by AstraZeneca. MT is employed at Statisticon for which AstraZeneca is a client. TJ, BS, DE and MJ report no conflict of interest relevant to this article. At the time this research was performed, DL was employed by Uppsala University, but has since been employed by AstraZeneca.

Patient consent for publication Not required.

Ethics approval The study was approved by the Stockholm regional ethics committee (registration number 2013/2206-31). The linkage of registers data was approved and performed by the Swedish National Board of Health and Welfare. Patients do not need to give consent for the use of public register data in Sweden. Provenance and peer review Not commissioned; externally peer reviewed.
Data sharing statement The data set supporting the conclusions of this article can be available upon request.

Open access This is an open access article distributed in accordance with the Creative Commons Attribution Non Commercial (CC BY-NC 4.0) license, which permits others to distribute, remix, adapt, build upon this work non-commercially, and license their derivative works on different terms, provided the original work is properly cited, appropriate credit is given, any changes made indicated, and the use is non-commercial. See: http://creativecommons.org/licenses/by-nc/4.0/.

\section{REFERENCES}

1. IDF Diabetes Atlas. 8th edn, 2017. Available: http://diabetesatlas.org/ resources/2017-atlas.html.

2. Vazzana N, Ranalli P, Cuccurullo C, et al. Diabetes mellitus and thrombosis. Thromb Res 2012;129:371-7.

3. Preis SR, Pencina MJ, Hwang SJ, et al. Trends in cardiovascular disease risk factors in individuals with and without diabetes mellitus in the Framingham Heart Study. Circulation 2009;120:212-20.

4. Bhatt DL, Eagle KA, Ohman EM, et al. Comparative determinants of 4-year cardiovascular event rates in stable outpatients at risk of or with atherothrombosis. JAMA 2010;304:1350-7.

5. Inzucchi SE, Bergenstal RM, Buse JB, et al. Management of hyperglycemia in type 2 diabetes, 2015: a patient-centered approach: update to a position statement of the American Diabetes Association and the European Association for the Study of Diabetes. Diabetes Care 2015;38:140-9.

6. International Diabetes Federation Guideline Development Group. Global guideline for type 2 diabetes. Diabetes Res Clin Pract 2014;104:1-52.

7. Giorda CB, Avogaro A, Maggini M, et al. Recurrence of cardiovascular events in patients with type 2 diabetes: epidemiology and risk factors. Diabetes Care 2008;31:2154-9.

8. Roger VL, Go AS, Lloyd-Jones DM, et al. Heart disease and stroke statistics-2011 update: a report from the American Heart Association. Circulation 2011;123:e18-e209.

9. Sardu C, Barbieri M, Balestrieri ML, et al. Thrombus aspiration in hyperglycemic ST-elevation myocardial infarction (STEMI) patients: clinical outcomes at 1-year follow-up. Cardiovasc Diabetol 2018;17:152.

10. Marfella R, Rizzo MR, Siniscalchi M, et al. Peri-procedural tight glycemic control during early percutaneous coronary intervention upregulates endothelial progenitor cell level and differentiation during acute ST-elevation myocardial infarction: effects on myocardial salvage. Int J Cardiol 2013;168:3954-62.

11. Marfella R, Sardu C, Balestrieri ML, et al. Effects of incretin treatment on cardiovascular outcomes in diabetic STEMI-patients with culprit obstructive and multivessel non obstructive-coronary-stenosis. Diabetol Metab Syndr 2018;10:10:1.

12. Jernberg T, Hasvold P, Henriksson M, et al. Cardiovascular risk in post-myocardial infarction patients: nationwide real world data demonstrate the importance of a long-term perspective. Eur Heart $J$ 2015;36:1163-70.

13. Wallerstedt SM, Wettermark B, Hoffmann M. The First Decade with the Swedish Prescribed Drug Register - A Systematic Review of the Output in the Scientific Literature. Basic Clin Pharmacol Toxicol 2016;119:464-9.

14. Bourdin A, Molinari N, Vachier I, et al. Mortality: a neglected outcome in OCS-treated severe asthma. Eur Respir J 2017;50:1701486.

15. Ludvigsson JF, Andersson E, Ekbom A, et al. External review and validation of the Swedish national inpatient register. BMC Public Health 2011;11:450.

16. Norhammar A, Bodegård J, Nyström T, et al. Incidence, prevalence and mortality of type 2 diabetes requiring glucose-lowering treatment, and associated risks of cardiovascular complications: a nationwide study in Sweden, 2006-2013. Diabetologia 2016;59:1692-701.

17. Rawshani A, Rawshani A, Franzén S, et al. Mortality and cardiovascular disease in type 1 and type 2 diabetes. N Engl J Med 2017;376:1407-18

18. Rawshani A, Rawshani A, Franzén S, et al. Risk factors, mortality, and cardiovascular outcomes in patients with type 2 diabetes. N Engl $J$ Med 2018;379:633-44.

19. Bethel MA, Patel RA, Merrill P, et al. Cardiovascular outcomes with glucagon-like peptide-1 receptor agonists in patients with type 2 diabetes: a meta-analysis. Lancet Diabetes Endocrinol 2018;6:105-13.

20. Marso SP, Daniels GH, Brown-Frandsen K, et al. Liraglutide and Cardiovascular Outcomes in Type 2 Diabetes. N Engl J Med 2016;375:311-22. 
21. Zinman B, Wanner C, Lachin JM, et al. Empagliflozin, Cardiovascular Outcomes, and Mortality in Type 2 Diabetes. N Engl J Med 2015;373:2117-28

22. Marso SP, Bain SC, Consoli A, et al. Semaglutide and Cardiovascular Outcomes in Patients with Type 2 Diabetes. N Engl J Med 2016;375:1834-44.

23. Huo L, Magliano DJ, Rancière F, et al. Impact of age at diagnosis and duration of type 2 diabetes on mortality in Australia 1997-2011. Diabetologia 2018;61:1055-63.

24. Hasvold LP, Bodegård J, Thuresson M, et al. Diabetes and CVD risk during angiotensin-converting enzyme inhibitor or angiotensin II receptor blocker treatment in hypertension: a study of 15,990 patients. J Hum Hypertens 2014;28:663-9.

25. Guðbjörnsdóttir SEB, Cederholm J, Zethelius B, et al. Swedish National Diabetes Register Annual report 2013. 2013. https://www. ndr.nu/pdfs/Annual_Report_NDR_2013.pdf (cited 05 Feb 2019).

26. Steinarsson AO, Rawshani A, Gudbjörnsdottir S, et al. Short-term progression of cardiometabolic risk factors in relation to age at type 2 diabetes diagnosis: a longitudinal observational study of
100,606 individuals from the Swedish National Diabetes Register. Diabetologia 2018;61:599-606.

27. Rawshani A, Svensson AM, Zethelius B, et al. Association between socioeconomic status and mortality, cardiovascular disease, and cancer in patients with type 2 diabetes. JAMA Intern Med 2016;176:1146-54.

28. Connolly V, Unwin N, Sherriff P, et al. Diabetes prevalence and socioeconomic status: a population based study showing increased prevalence of type 2 diabetes mellitus in deprived areas. J Epidemiol Community Health 2000;54:173-7.

29. Gambardella J, Sardu C, Sacra C, et al. Quit smoking to outsmart atherogenesis: Molecular mechanisms underlying clinical evidence. Atherosclerosis 2017:257:242-5.

30. Sardu C, Pieretti G, D'Onofrio N, et al. Inflammatory cytokines and SIRT1 levels in subcutaneous abdominal fat: relationship with cardiac performance in overweight pre-diabetics patients. Front Physiol 2018;9:1030.

31. Agardh E, Allebeck P, Hallqvist J, et al. Type 2 diabetes incidence and socio-economic position: a systematic review and metaanalysis. Int J Epidemiol 2011;40:804-18. 